\title{
Double Insertion of Thiophene Rings in Polyynediyl Chains To Stabilize Nano-scaled Molecular Wires with [Cp*(dppe)Fe] Termini
}

Séverine Roué, ${ }^{\&}$ Hiba Sahnoune, ${ }^{\&, \S}$ Loïc Toupet, ${ }^{\&}$ Jean-François Halet, ${ }^{\&} *$ and Claude Lapinte $^{\& *}$

\&Institut des Sciences Chimiques de Rennes, UMR 6226 CNRS-Université de Rennes 1, F35042 Rennes, France

${ }^{\S}$ Département de Chimie, Faculté des Sciences, Université M’Hamed Bougara, Algérie

\section{Supporting Information}

Including:

Page

1. Table S1. Crystallographic Data, Details of Data Collection and Structure Refinement Parameters for Compounds 3

2. Table S2. Computed (in brackets) and Experimental ESR Parameters for $\mathbf{2}^{+}$and $\mathbf{3}^{+}$

3. Figure S1. ${ }^{57} \mathrm{Fe}$ Mössbauer Spectrum for $\mathbf{2}\left(\mathbf{P F}_{\mathbf{6}}\right)$ at $80 \mathrm{~K}$.

4. Figure S2. UV-vis Spectra for 2, 3, 4, and $\mathbf{6}$

5 Figure S3. UV-vis Spectra for $\mathbf{3}\left(\mathbf{P F}_{\mathbf{6}}\right)_{\mathbf{n}}(n=0-2)$

6 Figure S4. Mulliken Atomic Spin Densities for $\mathbf{2}^{+}$ 
Table S1. Crystallographic data, details of data collection and structure refinement parameters for compound $\mathbf{3}$.

\begin{tabular}{|c|c|}
\hline & 3 \\
\hline Empirical Formula & $\mathrm{C}_{88} \mathrm{H}_{82} \mathrm{Fe}_{2} \mathrm{P}_{4} \mathrm{~S}_{2}$ \\
\hline Formula mass, $\mathrm{g} \mathrm{mol}^{-1}$ & 1439.23 \\
\hline Collection $\mathrm{T}, \mathrm{K}$ & $120(2)$ \\
\hline crystal system & Triclinic \\
\hline space group & $P-1$ \\
\hline$a(\AA)$ & $11.81270(10)$ \\
\hline$b(\AA)$ & $14.3695(2)$ \\
\hline$c(\AA)$ & $23.0200(3)$ \\
\hline$\alpha\left(^{\circ}\right)$ & $96.0470(10)$ \\
\hline$\beta\left(^{\circ}\right)$ & $103.9280(10)$ \\
\hline$\gamma\left({ }^{\circ}\right)$ & $103.9400(10)$ \\
\hline$V\left(\AA^{3}\right)$ & $3625.14(8)$ \\
\hline$Z$ & 2 \\
\hline$D_{\text {calcd }}\left(\mathrm{g} \mathrm{cm}^{-3}\right)$ & 1.319 \\
\hline Crystal size (mm) & $0.25 \times 0.22 \times 0.18$ \\
\hline$F(000)$ & 1508 \\
\hline abs coeff $\left(\mathrm{mm}^{-1}\right)$ & 0.593 \\
\hline$\theta$ range $\left(^{\circ}\right)$ & 2.329 to 26.999 \\
\hline range $h, k, 1$ & $0 / 15,-18 / 18,-29 / 29$ \\
\hline No. total refl. & 14829 \\
\hline No. unique refl. & 12174 \\
\hline Data/Restraints/Parameters & $12174 / 0 / 865$ \\
\hline Final $\mathrm{R}$ & $\mathrm{R}_{1}=0.0430$ \\
\hline$[I>2 \sigma(I)]$ & $\mathrm{wR}_{2}=0.1114$ \\
\hline $\mathrm{R}$ indices (all data) & $\mathrm{R}_{1}=0.0573$ \\
\hline & $\mathrm{wR}_{2}=0.1254$ \\
\hline Goodness of fit / F $F^{2}$ & 0.996 \\
\hline
\end{tabular}


Table 2-ESR. Computed (in brackets) and Experimental ESR Parameters for $\mathbf{2}^{+}$and $\mathbf{3}^{+}$

\begin{tabular}{llllll} 
Compd & $g_{1}$ & $g_{2}$ & $g_{3}$ & $g_{\text {iso }}$ & $\Delta g$ \\
$\mathbf{2 ( \mathbf { P F } _ { 6 } )}$ & 2.330 & 2.036 & 1.989 & 2.118 & 0.345 \\
& {$[2.093]$} & {$[2.020]$} & {$[1.999]$} & {$[2.037]$} & {$[0.094]$} \\
$\mathbf{3}_{\left(\mathbf{P F}_{6}\right)}$ & 2.335 & 2.034 & 1.986 & 2.128 & 0.379 \\
& {$[2.106]$} & {$[2.021]$} & {$[1.998]$} & {$[2.042]$} & {$[0.108]$} \\
\hline
\end{tabular}




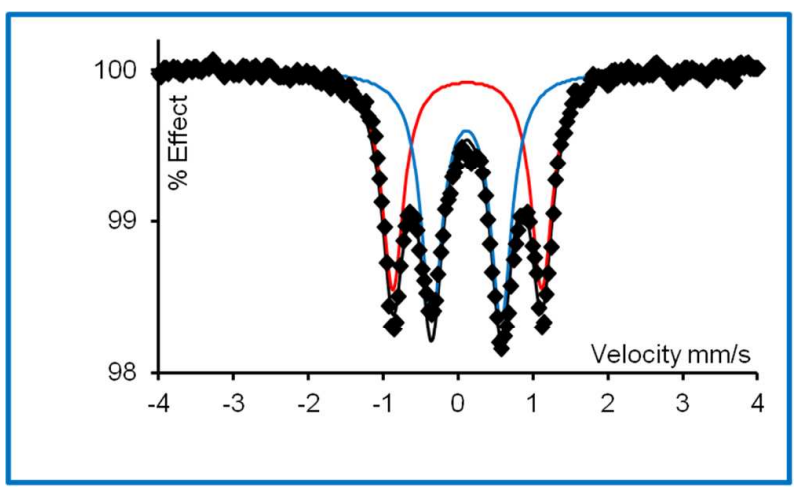

Figure S1. ${ }^{57} \mathrm{Fe}$ Mössbauer spectra for $\mathbf{2}\left(\mathbf{P F}_{\mathbf{6}}\right)$ at $80 \mathrm{~K}$ 


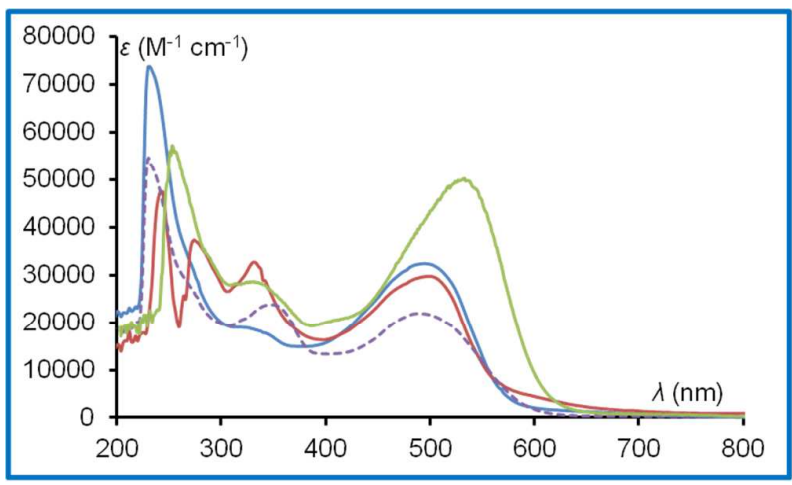

Figure S2. UV-vis spectra for 2 (blue), 3 (green), 4 (red), and $\mathbf{6}$ (purple) in $\mathrm{CH}_{2} \mathrm{Cl}_{2}$ 


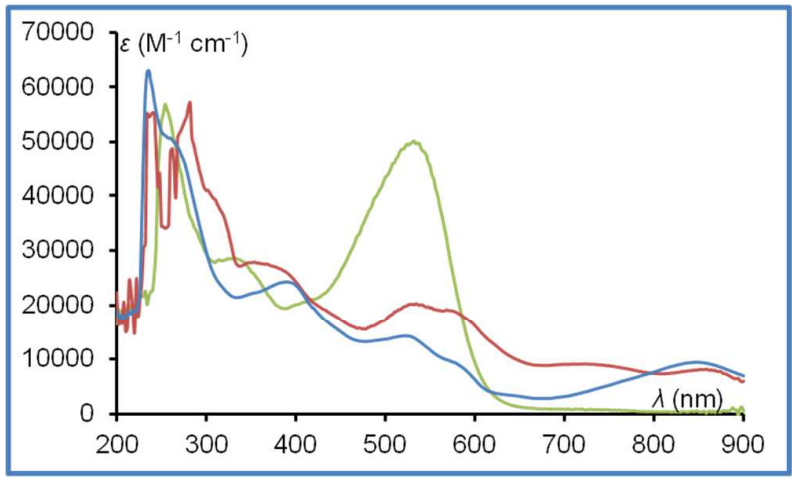

Figure S3. UV-vis for $\mathbf{3}\left(\mathbf{P F}_{\mathbf{6}}\right)_{\mathbf{n}}\left(\mathrm{n}=0\right.$ (green), $\mathrm{n}=1$ (red), $\mathrm{n}=2$ (blue)) in $\mathrm{CH}_{2} \mathrm{Cl}_{2}$ 


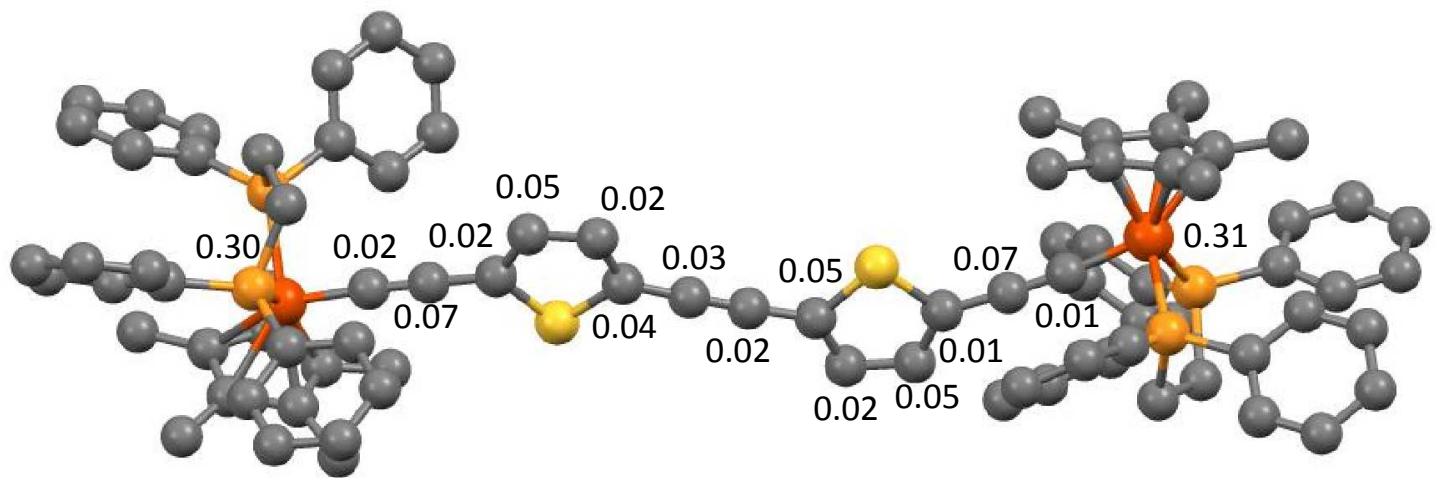

Figure S4. Mulliken Atomic Spin Densities for $\mathbf{2}^{+}$ 